\title{
Laparoscopic hepato-biliary-pancreatic surgery: present practices and prospects
}

\author{
Yongjun Chen, Renyi Qin, Xiaoping Chen (凶) \\ Department of General Surgery, Tongji Hospital, Tongji Medical College, Huazhong University of Science and Technology, Wuhan 430030, \\ China \\ (C) Higher Education Press and Springer-Verlag Berlin Heidelberg 2011
}

\section{Introduction}

Minimally invasive surgery was introduced in 1910, with the first laparoscopic cholecystectomy performed in 1987. Since then, laparoscopy has been embraced and adopted by every surgical specialty and has revolutionized modern surgical concepts. It represented an undisputed and significant advancement in the area of surgery [1]. The advantages of laparoscopic surgery include reduced pain, hospital stay, wound complications, recovery time, morbidity, and mortality. Initially, it was used principally as a diagnostic tool. Over the past 20 years, with enhancements in laparoscopic surgical instruments and increased experience with minimally invasive approaches, laparoscopic surgery has become widely accepted as a superior alternative to conventional open surgery in many medical fields. It has also been enthusiastically received by patients because of better cosmetic results. It has aided in the rapid development of colorectal cancer surgery and has achieved excellent initial results with enhanced postoperative recovery. More important, it has comparative oncological results as those from open resections.

In terms of hepato-biliary-pancreatic (HBP) surgery, however, laparoscopic approaches still remain a highly specialized field and have not been applied as quickly as for other organs. This is mainly due to limitations in instruments, anatomic complexities for poor exposure, risks of unexpectedly massive hemorrhage, difficulty of reconstruction after laparoscopic dissection, and iatrogenic injury caused by a narrow view field and the surgeon's blind spot. Although many reports have described laparoscopic partial liver resection, lateral segmentectomy, and distal pancreatectomy, laparoscopic major HBP surgery, such as hepatic lobectomy and pancreaticoduodenectomy, has not been widely developed. With rapid technological innovations, however, improved surgical techniques and accumulation of

Correspondence: chenxp@medmail.com.cn extensive experience by surgeons have improved the feasibility and safety of laparoscopic liver and pancreatic resections for properly selected patients. The indication of laparoscopic procedure is continuously expanding in the field of HBP surgery. In this editorial review, the indications, strengths, limitations, and prospects of the use of laparoscopic approaches in hepatic, biliary, and pancreatic diseases are explored.

\section{Laparoscopic liver surgery}

The application of laparoscopic approaches in hepatic surgery involves a lengthy process. In the early stage, only simpler hepatic procedures, such as de-roofing of symptomatic simple hepatic cysts, are well established and are commonly practised. Although laparoscopic liver resection (LHR) was first reported in the early 1990s, it is fully used in only a few expert centers worldwide. It remains uncommon for major liver resection partly because of the risk for massive hemorrhage. From this perspective, LHR represents one of the last limitations in the widespread use of laparoscopic surgery.

Tremendous progress has been achieved in the field of LHR. The remarkable advancement in surgical instrumentation for parenchyma dissection and ablative modalities has facilitated the development of alternative approaches involving multiple concomitant liver resections or concomitant thermal ablations. These techniques have been improved further with the introduction of laparoscopic techniques. When performed by experienced surgeons, laparoscopic techniques in liver resection are as safe as open techniques. The oncological results of laparoscopic surgery seem similar to or possibly even better than those of the open approach for hepatocellular carcinoma and colorectal and neuroendocrine liver metastases [2]. To date, indications for LHR have nearly equaled the classic indications for open liver resection [3,4]. These 
indications include benign (adenoma and symptomatic focal nodular hyperplasia, hemangioma or giant liver cysts) and malignant (both primary and metastatic) lesions in the liver and living donor liver harvesting. A high percentage of benign tumors has been presented in early series of LHR, whereas the proportion of malignant tumors has significantly increased in recent years. However, very large tumors or the necessity of biliary or vascular reconstruction has been considered the only ultimate contraindication to LHR [5].

One useful approach is hybrid liver resection which consists of hand-assisted laparoscopic resections and laparoscopic-assisted open liver. Hybrid liver resection refers to laparoscopic mobilization of the liver followed by hilar dissection and parenchymal transaction through open incision. A hand-port can be useful when the surgeon needs to have tactile control of resection, and it can be safely managed by hand-assisted techniques. Currently, the hand-assisted technique is mainly indicated in hepatectomies or resections of posterosuperior segments (segments I, VII, VIII, and IVa). These are considered challenging liver resections because they are technically difficult given poor exposure [6,7]. In addition, hybrid resection has been adopted especially for living donor right hepatectomies, enabling the use of the conventional surgical technique of liver handling together with the provision of a less invasive procedure [8]. Both hybrid techniques can present a bridge from open to total LHR.

With regard to complications, the perioperative mortality of LHR is reported to be below $1 \%$, which is the best result reported for open surgery. Its postoperative complication rate is around $10 \%-15 \%$ and is reported to be similar to or less than that observed in open liver resection. Postoperative bile leakage develops in about $1.5 \%$ of patients and represents a typical and crucial complication for LHR as it is for open surgery. Bile leakage is usually managed by percutaneous drainage and bile duct stenting. Postoperative intraabdominal bleeding is very rare but threatens complication; it can be managed via relaparoscopy. Although the reported liver resection-specific complications had similar rates with those of open surgery, the rate of liver decompensation among cirrhotic patients shows a decreasing trend with the application of the laparoscopic approach. General morbidity cardiopulmonary complications, abscesses, and wound complications are also reduced with the application of the laparoscopic approach. Further, the rate of incisional hernias after LHR is lower than that after open surgery [9].

\section{Laparoscopic biliary surgery}

Since the first laparoscopic cholecystectomy (LC) was performed in 1987, the biliary laparoscopic procedure has become a choice procedure, leading the progress in laparoscopic surgery. This advancement in turn facilitated the development of other procedures and provided a framework for education and training. Nowadays, LC is considered as the "gold standard" surgical procedure for cholelithiasis. Cholecystectomy previously required hospital admission for approximately a week. Now, it can be safely and reliably conducted laparoscopically as a day-case procedure. The indication for LC has widened to include conditions that were once considered its relative contraindications, such as acute cholecystitis, complicated cholecystitis, previous operation history, and old age. In addition, recent practices have established that single-incision laparoscopic cholecystectomy is safe, significantly reduces hospital stay, and is an acceptable alternative to the traditional multiport LC for some selected patients. Further, the use of singleincision laparoscopic approaches is continuously expanding to other areas [10]. The only issues related to LC are iatrogenic bile duct injuries associated with significant morbidity, mortality and a huge increase in care costs. Nevertheless, these issues have been significantly reduced with the accumulation of surgeons' experiences [11]. Moreover, with continuous improvement in technology and expertise in laparoscopic techniques, laparoscopic common bile duct exploration (CBDE) is becoming more popular and may be the next paradigm in the management of choledocholithiasis. Laparoscopic CBDE reduces inconvenience with its one-stage procedure in laparoscopic CBDE compared with a two-stage one in ERCP followed by laparoscopic cholecystectomy. Therefore, the risks of ERCP are reduced, which include hemorrhage, duodenal perforation, and pancreatitis. The results of laparoscopic CBDE are also comparable with those of the conventional twostage approach. Laparoscopic CBDE may also obviate the risk of the immediate and long-term problem of sphincterotomy of the ampulla of Vater, resulting in endoscopic extraction of the CBD stone. Therefore, laparoscopic CBDE is proven safe and effective, and issues have focused on comparisons between this form of management and orthodox endoscopic clearance. More important, pioneering operations of laparoscopic CBD/IHD exploration and Roux-en-Y choledochojejunostomy have been successfully performed. Consequently, this advancement has facilitated laparoscopic choledochal cyst excision for the treatment of congenital choledochal cyst type I. It is not only feasible but also highly effective in relieving biliary obstruction with good postoperative results in both benign and malignant conditions.

Bile tract malignant tumors including gallbladder carcinoma and extrahepatic cholangiocarcinoma (EHCC) consisting of hilar cholangiocarcinoma and 
upper bile duct and periampullary cancers are closely related to hepatic and/or pancreatic surgery. Therefore, the application of laparoscopic procedures in the biliary area depends substantially on the development of laparoscopic hepatic and pancreatic surgery. Due to lower R0 resection and more complications, the advantages of laparoscopy in bile tract malignant tumors may not be as great as initially expected. Laparoscopy may be employed in the management of patients with early forms of gallbladder cancer. Although the presence of adhesions may result in inadequate exploration, there is a subset of patients for whom the performance of a complete exam is possible. Further, laparoscopic lymphadenectomy and gallbladder bed resection are promising techniques in well-selected patients $[12,13]$. However, as to hilar cholangiocarcinoma and upper bile duct cancer, laparoscopic encircling of the hepatoduodenal ligament is proven difficult because of a narrow field of view and the surgeon's blind spot which may lead to unexpected bleeding or injury. Laparoscopy and, recently, endosonography seem to protect against unnecessary laparotomies in these patients. During the past 15 years, aggressive surgical approaches, including combined liver resections and vascular reconstructive surgical expertise, have improved survival in patients with EHCC. Laparoscopic resection is a potential alternative to open surgery for suitably selected patients with Bismuth type I hilar cholangiocarcinoma. For patients with type II tumors, the value of laparoscopic resection needs further evaluation. Notably, this conclusion has not been supported with an adequate amount of published data. There have also been no randomized controlled studies of both laparoscopic and conventional approaches.

\section{Laparoscopic pancreatic surgery}

Although laparoscopic surgery is evolving rapidly within general surgery, the development of laparoscopic pancreatic surgery has been slow and relatively appears to lag behind developments in other organs. Only a few centers have published their results on laparoscopic pancreatic surgery. The early reported outcomes for internal drainage of pancreatic pseudocysts, enucleation of benign insulinomas, and distal pancreatic resections are good; however, the experience is limited. A number of factors are assumed to contribute to this delay and have conspired against the development of an accepted laparoscopic approach and clear guidelines in pancreatic surgery. First, it is a technically demanding surgery involving long operating times, indirect access to retroperitoneal structures, dissection around major blood vessels, and construction of complex anastomoses. Given the friable nature of the pancreas, these factors are responsible for the high morbidity and frequent long hospital stays associated with pancreatic procedures, particularly if complications are involved. Second, localizing tumors in the pancreas usually requires tactile feedback to assess the site and size of the tumors. This capability is largely lost in laparoscopic surgery, so people have used laparoscopic ultrasound or hand-assisted techniques to examine the gland. Third, the learning curve is long, and these types of procedures require extensive experience in open pancreatic surgery combined with a high level of laparoscopic skill [14]. Considering that pancreatic carcinoma, which is suitable for presentation for a potentially curative procedure, is relatively uncommon, a slow progression along an already demanding learning curve is to be expected.

Despite these limitations, the theoretical advantages of laparoscopic pancreatic surgery include pain reduction and improved post-operative recovery, particularly reduced hospital stays, improved recovery, and the same oncological results as open surgery. Technological developments, particularly the improved availability of instruments to perform delicate anastomoses, also facilitated the use of advanced procedures in other fields, which are often directly applicable to pancreatic surgery. Combined with increased public healthcare expectations, these factors have helped drive the field forward.

To date, the areas that have attracted the most interest are diagnostic and staging of pancreas disease, enucleation of presumed benign or low-grade neuroendocrine lesions, and distal resections, although a small number of formal pancreaticoduodenectomies have been performed. Laparoscopic pancreatic exploration, including staging, biopsy, and palliation, has served as a powerful tool to assess local tumor extension and the presence or absence of peritoneal and serosal disease before potentially embarking on an extensive curative resection. When combined with steerable and sensitive ultrasound probes, the laparoscope has proven beneficial in assessing masses in the pancreas, involvement of local lymph nodes, extension of tumors to surrounding organs, and invasion of vascular structures $[15,16]$. It is particularly important for the assessment of cystic lesions in showing internal septae, mural nodules, and solid areas within cystic pancreatic tumors.

Laparoscopic enucleation of pancreatic lesions is usually performed for neuroendocrine tumors or benign cystic tumors without communication with the pancreatic duct [17]. Bleeding becomes minimal, there is no reconstruction required, and enucleation is associated with a low mortality rate. One area that remains a challenge, however, is the precise localization of tumors. Pre-operative assessment using all available modalities (CT, MRI, EUS, PET, and selective venous sampling) 
can generally locate single lesions accurately, but the finding must be confirmed intra-operatively. The majority of published series report no mortality, which is comparable to that of open procedures and the accepted standard for enucleation. Morbidity, however, showed an alarming increase to $66.6 \%$ and $77.8 \%$ in two published series. Fistula remains the main complication after laparoscopic enucleation at the rate of around $29 \%$ compared with $16 \%-23 \%$ in the best open series [18]. Recent technological developments and improved endoscopic procedures have substantially extended the applications of laparoscopic pancreatic resection. Laparoscopic distal pancreatectomy has gained rapid acceptance mainly because distal pancreatectomy, requiring no formal anastomosis of the pancreatic duct, is technically less demanding compared with right-sided resections. It also has the lowest reported intra-operative complications, mortality, and morbidity. Another reason for the higher rate of distal pancreatic resections is related to pathology because its benign or low-grade nature reduces concerns on resection margins and lymph node involvement.

Laparoscopic pancreaticoduodenectomy (LPD) remains the biggest challenge for pancreatic surgeons. This condition is due to technical difficulties not only in extensive dissection around major blood vessels but also in the formation of complex and multiple anastomoses, particularly pancreaticojejunostomy. No improvement in hospital stay, remarkably long operating times, and significantly higher mortality and morbidity rates are documented even when compared with open pancreaticoduodenectomy. To date, only limited series and case reports on laparoscopic procedures have been published, and LPD is not yet universally accepted as an alternative approach to open surgery. However, there is continued effort to continue the investigation of LPD with the aim of identifying and expanding indications and defining limitations. Major improvements are emerging despite the difficulty of laparoscopic reconstruction. Recent reports have described LPD as feasible and safe for selected patients, obtaining adequate surgical margins and lymphadenectomy. In the early period, the indication of LPD was limited only to benign or low-grade malignant diseases, such as intraductal papillary mucinous neoplasm and endocrine tumor. Recently, the indications for LPD have been extended to periampullary carcinoma, excluding invasive ductal carcinoma of the pancreas [19].

\section{References}

1. Jaffray B. Minimally invasive surgery. Arch Dis Child 2005; 90(5): 537-542

2. Farges O, Jagot P, Kirstetter P, Marty J, Belghiti J. Prospective assessment of the safety and benefit of laparoscopic liver resections. J Hepatobiliary Pancreat Surg 2002; 9(2): 242-248

3. Viganò L, Tayar C, Laurent A, Cherqui D. Laparoscopic liver resection: a systematic review. J Hepatobiliary Pancreat Surg 2009; 16(4): 410-421

4. Kazaryan AM, Røsok BI, Edwin B. Laparoscopic and open liver resection for colorectal metastases: different indications? HPB (Oxford) 2010; 12(6): 434, author reply 435

5. Cho A, Yamamoto H, Nagata M, Takiguchi N, Shimada H, Kainuma O, Souda H, Gunji H, Miyazaki A, Ikeda A, Tohma T, Matsumoto I. Laparoscopic major hepato-biliary-pancreatic surgery: formidable challenge to standardization. J Hepatobiliary Pancreat Surg 2009; 16 (6): 705-710

6. Cho JY, Han HS, Yoon YS, Shin SH. Feasibility of laparoscopic liver resection for tumors located in the posterosuperior segments of the liver, with a special reference to overcoming current limitations on tumor location. Surgery, 2008; 144:32-38

7. Marvin MR, Buell JF. Laparoscopic liver surgery. Adv Surg 2009; 43(1): 159-173

8. Koffron AJ, Kung R, Baker T, Fryer J, Clark L, Abecassis M. Laparoscopic-assisted right lobe donor hepatectomy. Am J Transplant 2006; 6(10): 2522-2525

9. Edwin B, Nordin A, Kazaryan AM. Laparoscopic liver surgery: new frontiers. Scand J Surg 2011; 100(1): 54-65

10. Joseph S, Todd Moore B, Brent Sorensen G, Earley JW, Tang F, Jones P, Brown KM. Single-incision laparoscopic cholecystectomy: a comparison with the gold standard. Surg Endosc 2011;25 (9):3008-3015 [Epub ahead of print] DOI: 10.1007/s00464-0111661-x

11. Karvonen J, Gullichsen R, Laine S, Salminen P, Grönroos JM. Bile duct injuries during laparoscopic cholecystectomy: primary and long-term results from a single institution. Surg Endosc 2007; 21(7): 1069-1073

12. Tang CN, Siu WT, Ha JP, Tai CK, Tsui KK, Li MK. Laparoscopic biliary bypass - a single centre experience. Hepatogastroenterology 2007; 54(74): 503-507

13. de Aretxabala X, Leon J, Hepp J, Maluenda F, Roa I. Gallbladder cancer: role of laparoscopy in the management of potentially resectable tumors. Surg Endosc 2010; 24(9): 2192-2196

14. Al-Taan OS, Stephenson JA, Briggs C, Pollard C, Metcalfe MS, Dennison AR. Laparoscopic pancreatic surgery: a review of present results and future prospects. HPB (Oxford) 2010; 12(4): 239-243

15. Ahmed SI, Bochkarev V, Oleynikov D, Sasson AR. Patients with pancreatic adenocarcinoma benefit from staging laparoscopy. J Laparoendosc Adv Surg Tech A 2006; 16(5): 458-463

16. Gaujoux S, Allen PJ. Role of staging laparoscopy in peri-pancreatic and hepatobiliary malignancy. World J Gastrointest Surg 2010; 2(9): 283-290

17. Crippa S, Bassi C, Salvia R, Falconi M, Butturini G, Pederzoli P. Enucleation of pancreatic neoplasms. Br J Surg 2007; 94(10): 12541259

18. Fernández-Cruz L, Blanco L, Cosa R, Rendón H. Is laparoscopic resection adequate in patients with neuroendocrine pancreatic tumors? World J Surg 2008; 32(5): 904-917

19. Kendrick ML, Cusati D. Total laparoscopic pancreaticoduodenectomy: feasibility and outcome in an early experience. Arch Surg 2010; 145(1): 19-23 